\title{
La educación en derechos humanos y el currículo oculto: consideraciones teórico-prácticas sobre el cotidiano escolar brasileño
}

\author{
ALIA BARRIOS GONZÁLEZ* \\ Universidad de Brasilia - Brasil \\ Recibido el 08-10-18; primera evaluación el 08-11-19; \\ segunda evaluación el 12-12-19; aceptado el 23-01-20
}

\section{RESUMEN}

Este artículo presenta consideraciones teórico-prácticas sobre la educación en derechos humanos (EDH), en el contexto de actividades pedagógicas de la Enseñanza Básica brasileña, considerando sus directrices y las interacciones/ relaciones sociales de la escuela (currículo oculto). Con base en una perspectiva sociocultural constructivista, presentamos el análisis microgenético de situaciones escolares que surgieron en una investigación realizada en una institución de enseñanza pública en Brasilia, Distrito Federal. En el contexto de una actividad sobre derechos humanos, surgen desafíos enfrentados por la escuela contemporánea con relación a la implementación de la EDH. Es necesario desarrollar una pedagogía participativa que haga de la EDH un elemento relevante para los alumnos, y también una formación docente que parta del análisis de las prácticas pedagógicas y de las acciones programáticas de la EDH.

Palabras clave: educación en derechos humanos, currículo oculto, Educación Básica.

\footnotetext{
* Graduada en Psicología por la Universidad de La Habana, Cuba. Diploma revalidado por el Instituto de Psicología de la Universidad de Brasilia (UNB), Brasil. Magíster en Psicología por el Programa de Posgrado en Desarrollo Humano y Salud del Instituto de Psicología de la UNB. Doctora en Psicología por el Programa de Posgrado en Desarrollo Humano y Salud del Instituto de Psicología de la UNB. Profesora adjunta del área de Psicología de la Educación, en el Departamento de Teoría y Fundamentos (TEF), de la Facultad de Educación (FE), de la UNB. Profesora del Programa de Posgrado en Educación Modalidad Profesional (PPGE-MP) de la Facultad de Educación (FE), de la UNB. Correo electrónico: aliabarrios@gmail.com
} 


\section{Human rights education and the hidden curriculum: Theoretical and practical considerations on the Brazilian school routine}

\section{Abstract}

This paper presents theoretical and practical considerations about human rights education (HRE), in the context of pedagogical activities of Brazilian Basic Education, considering its guidelines and the school's social interactions/ relationships (hidden curriculum). Based on a sociocultural constructivist theoretical approach, we present the microgenetic analysis of school situations that emerged in a research carried out in a public educational institution in Brasília, Distrito Federal. In the context of a human rights activity, the challenges faced by the contemporary school in relation to the actual implementation of HRE arise. It is necessary to develop a participatory pedagogy that makes HRE a relevant element for the students, as well as a teacher formation anchored in the analysis of the pedagogical practices and the programmatic actions of the HRE.

Keywords: Human Rights Education, hidden curriculum, Basic Education.

\section{A educaçáo em direitos humanos e o currículo oculto: consideraçóes teórico- práticas sobre o cotidiano escolar brasileiro}

\section{RESUMO}

Este artigo apresenta consideraçóes teórico-práticas sobre a educação em direitos humanos (EDH), no contexto de atividades pedagógicas do Ensino Básico brasileiro, considerando suas diretrizes e as interaçóes/relaçóes sociais da escola (currículo oculto). Com base em uma perspectiva sociocultural construtivista, apresentamos a análise microgenética de situaçóes escolares que surgiram em uma pesquisa realizada em uma instituição pública de ensino em Brasília, Distrito Federal. No contexto de uma atividade sobre direitos humanos, surgem desafios enfrentados pela escola contemporânea em relação à implementação da EDH. É necessário desenvolver uma pedagogia participativa que torne a $\mathrm{EDH}$ um elemento relevante aos alunos, assim como uma formação docente ancorada na análise das práticas pedagógicas e das açóes programáticas da EDH.

Palavras-chave: educação em direitos humanos, currículo oculto, Ensino Básico. 


\section{INTRODUCCIÓN}

Este artículo tiene como objetivo principal proponer consideraciones teóricoprácticas sobre la educación en derechos humanos $(\mathrm{EDH})$, en el contexto de las actividades pedagógicas, características de la Enseñanza Básica brasileña, considerando sus directrices y las interacciones/relaciones sociales (currículo oculto) que posibilitan, o no, los objetivos pautados para el currículo manifiesto.

Datos de diferentes investigaciones muestran que las acciones pedagógicas para la formación de valores sociales fundamentales para los derechos humanos $(\mathrm{DDHH})$ permanecen en un segundo plano delante de aspectos como la organización de la rutina y de las actividades, la disciplina y la transmisión unilateral de conocimientos (Barrios, 2009, 2013; Branco, Barreto y Barrios, 2018; Lima, 2000, 2017; Menin, 2002). Aunque ya se hayan implementado acciones importantes en el contexto de la $\mathrm{EDH}$, todavía son necesarias nuevas estrategias pedagógicas y de formación inicial y continua de los/as docentes que posibiliten cambios en el posicionamiento de los educadores delante de los procesos de enseńanza y aprendizaje. En el contexto de la $\mathrm{EDH}$, los métodos pedagógicos deben estimular la participación activa de todos los actores del escenario escolar, así como el análisis crítico y la aplicación de los DDHH en la vida cotidiana, de acuerdo con los documentos y directrices presentados a seguir.

Diversos documentos internacionales abordan la EDH. Entre esos documentos se destaca el Programa Mundial para la Educación en Derechos Humanos, aprobado por la ONU en 2004, que define la EDH como un conjunto de actividades educativas, de capacitación y de difusión de la información que visan la propagación de conocimientos sobre los DDHH, la creación de mecanismos para protegerlos y la trasmisión de actitudes necesarias para promover, defender y aplicar los DDHH en la vida cotidiana. Algunos de los principios conductores de la $\mathrm{EDH}$ son las siguientes: (a) promover la interdependencia, la indivisibilidad y la universalidad de los derechos humanos; (b) fomentar el respeto y la valorización de las diferencias; (c) utilizar métodos pedagógicos participativos; (d) fomentar ambientes de enseñanza-aprendizaje que estimulen la participación, el goce de los derechos humanos y el desarrollo pleno de la personalidad/individualidad humana (Unesco, 2012).

En el contexto de los sistemas educacionales, el Programa Mundial para la Educación en Derechos Humanos resalta que la EDH debe ser entendida como parte integrante del derecho a la educación, la cual debe tener como objetivo central la preparación de la persona para la vida cotidiana, así como el fortalecimiento de sus capacidades para disfrutar de todos los DDHH 
y fomentar una cultura permeada por valores sociales apropiados para los mismos. Ese tipo de educación debe ser ofrecida a todas las personas y especialmente a los niños, como una herramienta indispensable para conseguir el respeto de sus derechos delante de los desafíos impulsados por la mundialización, por las nuevas tecnologías y por fenómenos conexos (Unesco, 2012).

Siendo así, la EDH debe aparecer en dos dimensiones en todo sistema educacional. La primera dimensión se refiere a la presencia de los contenidos de los DDHH en todos los elementos primordiales para el proceso de enseńanza-aprendizaje. Los planes de enseńanza/estudio, los materiales didácticos y los métodos pedagógicos y de capacitación deben conducir al aprendizaje de los DDHH. Esta dimensión es definida por el Programa Mundial para la Educación en Derechos Humanos como «los derechos humanos en el contexto educativo». La segunda dimensión se refiere a la práctica de los DDHH en los diferentes espacios educativos, por parte de todos sus actores, y es definida por el programa como «la realización de los derechos humanos en la educación».

Con base en esos presupuestos, la EDH en la educación primaria y secundaria debe incluir: (a) las políticas, leyes y estrategias de educación coherentes con los DDHH; (b) la implementación de políticas que faciliten la participación de todos los interesados; (c) el ambiente de aprendizaje que debe ofrecer oportunidades para que todos los actores del contexto educacional (estudiantes, profesores, funcionarios, administradores y padres) practiquen los DDHH en las actividades de la vida real; (d) todos los procesos e instrumentos de enseńanzaaprendizaje que deben ser fundamentados en los derechos; (e) la educación y el desarrollo profesional de los profesores y otros profesionales (Unesco, 2012).

En el contexto educacional brasileńo, partiendo del Programa Mundial para la Educación en Derechos Humanos, diversos documentos de carácter nacional definen directrices fundamentales para la EDH: el Plan Nacional de Educación en Derechos Humanos, la resolución 1 del 30 de mayo de 2012 (Poder Executivo do Brasil, 2012), y las Directrices Curriculares Nacionales para la Educación Básica de 2013 (Ministério da Educação et al., 2013).

Esta resolución 1 del 30 de mayo de 2012 (Resolución CNE/CP 1/2012), que establece las Directrices Nacionales para la Educación en Derechos Humanos, define la misma como un proceso sistemático y multidimensional, orientador de la formación integral de los sujetos de derecho, y articulado con: (a) la aprehensión de conocimientos históricamente construidos sobre los $\mathrm{DDHH}$; (b) la afirmación de valores, actitudes y prácticas sociales que expresen la cultura de los DDHH; (c) la formación de una consciencia ciudadana; (d) el desarrollo de procesos metodológicos participativos y de construcción colectiva; (e) el fortalecimiento de prácticas que generen acciones e instru- 
mentos a favor de la promoción, de la protección y defensa de los DDHH (Poder Executivo do Brasil, 2012, sección 1, p. 48).

Las Directrices Curriculares Nacionales para la Educación Básica brasileña (Ministério da Educação et al., 2013) resaltan el trabajo con los DDHH como tema transversal del currículo, en función de su complejidad, de su presencia intensa en los diversos sistemas sociales (comunidad, familia y escuela), y por su existencia explícita e implícita en las diversas áreas del currículo (Ministério da Educação et al., 2013). De acuerdo con las directrices, todas las áreas del conocimiento abarcan asuntos importantes para los DDHH, pero esos temas no son suficientes para explicarlos de manera amplia. Una vez que los contenidos de los DDHH están relacionados con la formación personal del individuo, también lo están con el desarrollo de un conjunto de creencias, valores y normas morales y éticas compartidas en los diferentes contextos socioculturales donde cada individuo actúa y se desarrolla. Siendo así, la transversalidad se refiere a la posibilidad de establecer, en la práctica educativa, una relación entre aprender conocimientos teóricamente sistematizados de las diferentes áreas y las cuestiones de la vida real y de su transformación. Para sistematizarla, es necesario incluir en la organización curricular temas relacionados a los DDHH, la ética, la pluralidad cultural, etc. En principio, la transversalidad puede garantizar un trabajo continuo y profundo con los conocimientos de diversas áreas y las situaciones problema que surgen cotidianamente.

\section{Marco teórico}

Analizando los diferentes documentos internacionales y nacionales sobre la $\mathrm{EDH}$, es posible decir que los mismos ofrecen directrices fundamentales para la consolidación de la EDH en el currículo manifiesto, entendido como un constructo técnico que orienta procedimientos, métodos y técnicas educativas, y que posibilita que la escuela alcance sus objetivos. Entre tanto, esa inclusión en la organización curricular no es suficiente. La EDH no solo implica un cambio en los objetivos de la educación, sino que también implica un cambio en el posicionamiento de los educadores delante del proceso de enseñanzaaprendizaje y un cambio en la visión que muchos educadores tienen sobre el papel de la escuela con relación a la formación de creencias y valores éticos y morales (Tavares, 2007).

Con relación a esa cuestión, la perspectiva sociocultural constructivista enfatiza que es necesario que los educadores dejen de lado el modelo de transmisión unidireccional del conocimiento, que parte de la existencia de contenidos trasmitidos entre generaciones y asimilados por la persona de forma pasiva. 
Es importante que el espacio educativo deje de ser un espacio de reproducción de normas, reglas, creencias y valores establecidos sin la posibilidad de protagonismo por parte de nińos y jóvenes. Asimismo, debe asumir su papel fundamental en el desarrollo y educación de valores sociales y morales del individuo, papel que ha sido colocado como una tarea de la familia por excelencia (Barrios, 2009, 2013; Koga y Rosso, 2016; Lima, 2000, Menin, 2002; Silva y Menin, 2015).

En el contexto de la EDH, los educadores deben asumir la enseńanza y el aprendizaje como procesos de construcción y resignificación bidireccional de conocimientos, experiencias y valores; es decir, deben partir de la idea de que todos los interlocutores del proceso educativo son coconstructores de nuevos elementos socioculturales. Todos los participantes de los procesos de enseñanza y aprendizaje tienen la posibilidad de construir novedades a partir de sus papeles sociales, de sus experiencias personales y de sus conocimientos previos (Valsiner, 1989, 2007, 2012).

En la práctica pedagógica cotidiana, eso significa fomentar y construir relaciones e interacciones sociales con base en el respeto y en la valorización de la diversidad, entendiendo que esas relaciones e interacciones son el escenario pedagógico fundamental de la EDH. Conforme a lo seńalado por varios autores de perspectivas socioculturales (Ratner, 2002; Rogoff, 2005; Valsiner, 1989, 2007, 2012; Vygotsky, 1984, 2004), las relaciones e interacciones sociales se constituyen el contexto principal, en el cual se internalizan y externalizan las creencias y valores sociales compartidos en nivel colectivo. Esa internalización y externalización tienen carácter singular y envuelven significados importantes para cada sujeto, de acuerdo con sus vivencias e historia de vida.

Entre tanto, varias investigaciones realizadas en el contexto educativo brasileño con base en la perspectiva sociocultural constructivista (Barreto, 2004; Barrios, 2009; 2013; Lima, 2000, 2017; Manzini, 2013; Palmeire, 2003; Salomão, 2001) señalan que los diversos significados socioculturales que se construyen y reconstruyen en las relaciones e interacciones sociales del espacio escolar hacen parte del currículo oculto, entendido como un conjunto de factores que no son programados en un sentido pedagógico, pero que pueden influenciar significativamente las experiencias de los alumnos en su rutina escolar (Vallance, 1983). El currículo oculto puede ser desconocido para la mayoría de los educadores y abarca lo siguiente: los patrones de interacciones que se estableen entre los profesores y sus alumnos, las expectativas de los educadores en relación con el proceso de enseñanza-aprendizaje, la estructura de las situaciones de aprendizaje (actividades y rutina), y las reglas y valores implícitos que permiten que esa estructura se mantenga (DeVries y Zan, 1998, 2003; Branco, 1989, 2003; 
Branco y Mettel, 1995; Vallance, 1983). Resumiendo, el currículo oculto abarca la función socializadora de la escuela, la cual es muchas veces ignorada por los profesores, lo que puede ocasionar conflictos no intencionales entre los objetivos del currículo manifiesto y las acciones del cotidiano (Barrios, 2009, 2013; Tappan, 1992, 1997, 1998; Palmieri, 2003; Salomão, 2001).

Una vez que la $\mathrm{EDH}$ necesita de coherencia entre las prácticas pedagógicas y sus valores intrínsecos conforme a los significados y resignificados en las interacciones del cotidiano, consideramos fundamental analizar y comprender cómo los contenidos y valores relacionados a los DDHH se presentan tanto en el currículo oculto como en el currículo manifiesto. De acuerdo con Magendzo (2006), es importante crear un ambiente propicio para que exista un aprendizaje significativo de las actitudes y valores fundamentales para la construcción de una cultura de derechos humanos.

Partiendo de las ideas anteriores y con el objetivo de ilustrar brevemente la importancia y el efecto del currículo oculto, presentamos el análisis de situaciones escolares específicas que surgieron en una investigación sobre el desarrollo moral en la Enseñanza Primaria brasileña.

\section{Metodología}

El estudio fue realizado en una escuela pública de Educación Primaria del Distrito Federal. Los datos, colectados en 2013, son parte de un proyecto de investigación mayor de carácter longitudinal y en ejecución, que objetiva mapear la implementación de las directrices de la $\mathrm{EDH}$ en escuelas de Educación Primaria en el Distrito Federal. Con base en la perspectiva teórico-metodológica sociocultural constructivista, la investigación tuvo como objetivo central identificar aspectos relevantes para el desarrollo moral de los alumnos a partir del análisis del discurso, de la argumentación y de la dinámica de relaciones de un grupo de alumnos de quinto grado y de su profesor. Siendo así, participaron del estudio los 22 alumnos del grupo de quinto grado (12 alumnas y 10 alumnos) y su profesor con 15 años de experiencia docente y formación en Pedagogía. El profesor fue seleccionado para participar del estudio en función de su experiencia con métodos pedagógicos participativos, su formación complementaria e interés por el tema.

Fueron realizadas ocho sesiones de observación naturalista de diversas actividades pedagógicas del grupo. Esas sesiones de observación directa fueron registradas en un diario de campo, siguiendo un protocolo de registro y análisis de observación construido específicamente para la investigación. En las ocho sesiones de observación naturalista, participaron los 22 alumnos del grupo 
y el profesor. Esas sesiones de observación tuvieron carácter etnográfico y posibilitaron un conocimiento previo sobre las actividades pedagógicas e interacciones características del grupo.

Después de las sesiones de observación directa, fueron realizadas dos sesiones de observación estructurada, filmadas y posteriormente transcritas para el protocolo de registro y análisis. Para la realización de una de las dos sesiones de observación estructurada el profesor fue invitado a planificar una actividad con el objetivo de abrir un espacio de discusión sobre cuestiones morales entre los alumnos a partir de temas sugeridos por la investigadora: autoridad, conflictos, obediencia, derechos humanos, autonomía, prejuicios y diversidad. El profesor seleccionó el tema de los derechos humanos por ser un tema «... muy importante para todos. Desde niños, todos tenemos que saber nuestro derechos y deberes».

La actividad realizada por el profesor tuvo 44 minutos y 37 segundos de duración y contó con la participación de 19 alumnos (10 niñas y 9 nińos). En el primer momento de la actividad, el grupo fue dividido en pequeños grupos (3 de niñas y 3 de niños) que leyeron, discutieron y elaboraron un texto y dibujo sobre uno de los artículos de la Declaración Universal de los Derechos Humanos (DUDH). El texto y el dibujo elaborados por cada grupo fueron registrados en un protocolo de registro confeccionado por el profesor específicamente para la actividad. Después de la discusión y producción gráfica en grupos pequeños, todos los participantes formaron una rueda para socializar sus discusiones y producciones, y para conversar sobre el tema de manera general. En el presente trabajo partimos de esa actividad sobre el tema de los $\mathrm{DDHH}$ para proponer consideraciones teórico-prácticas sobre la EDH en el contexto de las actividades pedagógicas.

La observación de la actividad planificada por el profesor fue analizada microgenéticamente en función de la importancia y adecuación del análisis microgenético para el estudio de las interacciones sociales. Según Kelman y Branco (2004), el análisis microgenético permite analizar la comunicación como un proceso dinámico, destacando el papel igualmente importante de todos los participantes. Ese tipo de análisis también destaca la interdependencia existente entre los individuos y los diferentes contextos socioculturales en que ellos están inmersos. Las autoras enfatizan que, en el contexto de la microgénesis, la función comunicativa del lenguaje pasa a tener un papel central. Esa función comunicativa debe ser entendida en una perspectiva dialógica y bidireccional, una vez que las interacciones comunicativas de los participantes pueden posibilitar transformaciones en los significados socioculturales compartidos. 
En el primer momento del análisis microgenético de la actividad estructurada, fue realizada una observación y análisis previo de la sesión filmada, identificando los fragmentos más significativos para el estudio y destacando el tiempo y continuidad de las interacciones. Los fragmentos seleccionados para el análisis están relacionados con las reflexiones y colocaciones de los niños sobre los diferentes artículos de la DUDH, destacando sus creencias y valores, y la forma como los mismos fueron discutidos, o no, con el grupo. Después de ese análisis inicial, la sesión fue transcrita integralmente y dividida en episodios que recibieron un título, considerando colocaciones importantes por parte de los niños y del profesor. Cada episodio delimitado agrupó un conjunto de interacciones continuas sobre cuestiones relevantes para el estudio dentro de un mismo tópico o tema. En los diferentes episodios, fueron utilizados, como unidades de análisis, pequeños fragmentos de los diálogos e interacciones entre los participantes de la actividad, con el objetivo de posibilitar un análisis minucioso de las interacciones y de las cuestiones agrupadas dentro de un mismo episodio. Fueron delimitados un total de seis episodios.

Para viabilizar la transcripción y posterior comprensión del protocolo de registro y análisis de la actividad, fue elaborada la siguiente leyenda:

- F (femenino) y M (masculino): Los nińos fueron identificados de acuerdo con el sexo y enumerados según el lugar que cada uno ocupó en la rueda de conversación, que fue la forma seleccionada por el profesor para conducir el inicio y término de la actividad. La enumeración de los niños comenzó por el primer nińo sentado a la derecha de la filmadora.

- Ns (niños): Indica que varios niños hablaron al mismo tiempo o respondieron juntos la misma pregunta.

- P (profesor): Indica las acciones del profesor.

... (puntos suspensivos): Indican pausa en el habla de los participantes.

$\rightarrow$ (flecha hacia la derecha): Indica el inicio del flujo de las interacciones.

(números entre paréntesis al inicio de cada fragmento): Indican las unidades de análisis del episodio.

\section{Resultados y Discusión}

El análisis microgenético de la actividad sobre el tema de los DDHH apuntó resultados importantes como el establecimiento de interacciones entre el profesor y sus alumnos (currículo oculto) que redujeron la posibilidad de participación activa de los segundos, presentando el conocimiento de forma unidireccional y asimétrica. Las interacciones establecidas también interfirieron 
en la consecución del objetivo principal de la actividad, que era promover un espacio de discusión sobre los DDHH. Para ilustrar los resultados, presentamos la transcripción y análisis de dos fragmentos (unidades de análisis) de la socialización de las discusiones y producciones de los pequeños grupos, sobre los artículos de la DUDH. Los dos fragmentos presentados hacen parte del Episodio VI, titulado «¡Felicidades! ¡Me gustó!», frases usadas por el profesor que se limitó a felicitar a los alumnos, sin abrir un espacio real de discusión sobre los artículos de la DUDH y las reflexiones de los niños. Siguen los fragmentos 5 y 7 del Episodio VI de la actividad estructurada sobre los DDHH:

Tabla 1. Protocolo de análisis microgenético del fragmento 5 del Episodio VI «¡Felicidades! ¡Me gustó!»

\begin{tabular}{|l|l|}
\hline (5) & $\begin{array}{l}\rightarrow \text { M4 se levanta de la silla con la hoja de } \\
\text { registro de la actividad en la mano. }\end{array}$ \\
\hline P: ¿Cuál es el artículo? & M4: 11 \\
\hline P: ¿Qué ustedes piensan sobre el artículo? & $\begin{array}{l}\text { M4: Nuestro grupo piensa que esa ley } \\
\text { está bien elaborada porque muchas } \\
\text { personas son acusadas inocentemente, } \\
\text { porque muchas personas son acusadas } \\
\text { por prejuicio o venganza. }\end{array}$ \\
\hline $\begin{array}{l}\text { P: ¡Muy bien! ¡Tú dijiste una palabra clave! } \\
\text { ¿Cuál fu esa palabra que tú dijiste? }\end{array}$ & M4 mira al profesor. \\
\hline $\begin{array}{l}\text { P: ¿Cuál fue la última palabra que tú dijiste? } \\
\text { Una palabra fundamental en eso que tú } \\
\text { dijiste... }\end{array}$ & \\
\hline $\begin{array}{l}\text { P: ¡Prejuicio! ¡No se puede tener prejuicios! } \\
\text { No se puede tener prejuicio en relación } \\
\text { a nada... creencia, raza, religión... por } \\
\text { futbol tampoco... aunque sea ‘flamengo’... } \\
\text { ¿Está bien? Entonces, todo el mundo tiene } \\
\text { el derecho de hacer lo que quiera siempre } \\
\text { que no moleste a los otros. ¿Está bien? Y el } \\
\text { prejuicio es algo que se ve mucho por ahí... y } \\
\text { es una cosa que tenemos que combatir. ¿Está } \\
\text { bien? ¿A cuál grupo le toca ahora? Grupo... }\end{array}$ & \\
\hline & \\
\hline
\end{tabular}




\section{Hoja de registro de la actividad del Grupo 4:}

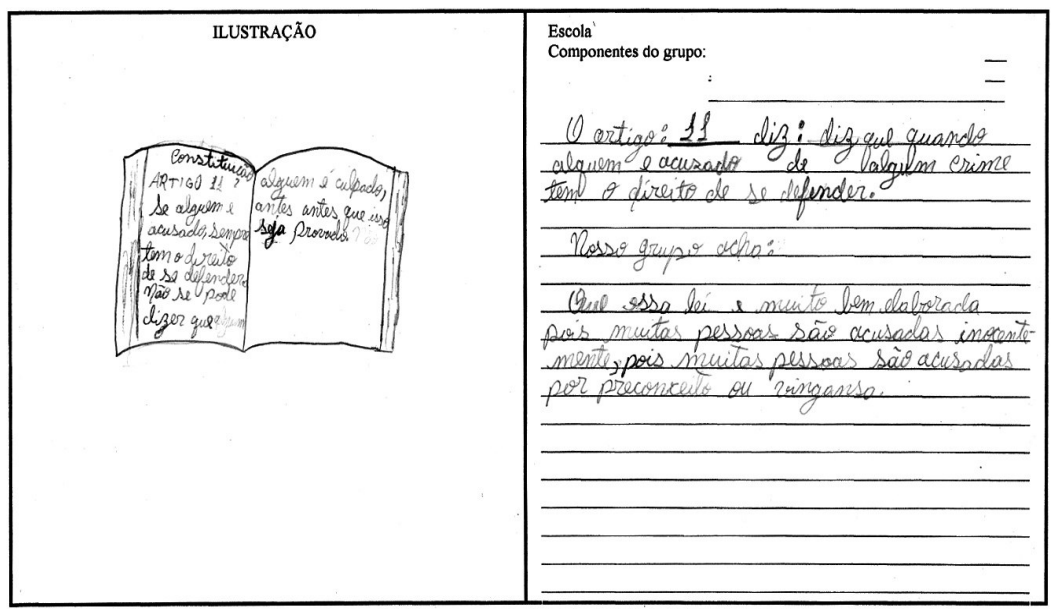

En este trecho impresiona la forma rápida de abordar el resultado de la tarea que fue dada al grupo. El profesor dice que tener prejuicios está errado y pasa de inmediato para el próximo grupo. Los nińos no fueron cuestionados en relación con lo que ellos entienden por prejuicio, ni sobre los tipos de prejuicio que existen en la sociedad brasileña. La reflexión realizada por el grupo de alumnos no fue aprovechada por el profesor para discutir con el grupo como un todo. El profesor se limitó a evaluar de manera simple la reflexión/presentación de los niños con la frase ‘Muy bien!', apuntando el término prejuicio como 'una palabra fundamental'.

En ningún momento el profesor les pide a los alumnos que citen ejemplos de prejuicios a partir de su experiencia o vida cotidiana. El profesor diserta brevemente contra el prejuicio, colocando entre sus ejemplos el futbol ( ${ }^{\prime} N o$ se puede tener prejuicios! No se puede tener prejuicio en relación a nada... por futbol tampoco... aunque sea 'Alamengo'... ¿Está bien?') y dejando de lado situaciones sociales relacionadas con el artículo analizado por el grupo. En vez de indagar las concepciones y creencias de los alumnos, colocó sus propias creencias sin explicarlas ('...y es una cosa que tenemos que combatir). Con esa intervención, el profesor no considera toda la complejidad de los procesos de construcción de los prejuicios y de los valores sociales. En el habla del profesor, los valores aparecen como algo que la persona decide tener o no, independientemente de todos los aspectos cognitivos y emocionales que abarcan. $\mathrm{O}$ sea, son resultados de una elección simple.

La producción escrita y la producción gráfica del grupo 4 mostraron una reflexión importante por parte de los niños en relación con el artículo 11, una vez que el artículo no se refiere a los prejuicios. Esas cuestiones colocadas por los niños no fueron exploradas por el profesor.

Este trecho muestra la disponibilidad concreta de los niños para reflexionar y realizar una discusión más amplia y autónoma sobre el tema propuesto, entre tanto, esa disponibilidad no fue aprovechada si no limitada por el adulto.

Fuente: Sesión de observación estructurada y filmada de la investigación. Elaboración propia. 
Tabla 2. Protocolo de análisis microgenético del fragmento 7 del Episodio VI «¡Felicidades! ¡Me gustó!»

\begin{tabular}{|c|c|}
\hline \multirow[t]{2}{*}{ (7) } & $\rightarrow$ F8 se levanta de la silla y mira al profesor. \\
\hline & $\begin{array}{l}\text { M5 se levanta de la silla con la mano en la } \\
\text { nariz, pasa por delante del profesor. }\end{array}$ \\
\hline \multirow[t]{2}{*}{$\begin{array}{l}\text { P: ¿Qué pasó? (Hablando con M5 que pasa } \\
\text { por delante de él.) }\end{array}$} & \\
\hline & $\begin{array}{l}\text { M5 hace un gesto con la mano, indicando } \\
\text { que va a salir del aula. }\end{array}$ \\
\hline \multirow[t]{2}{*}{ P: Ve allá fuera y regresa rápido... } & \\
\hline & M5 sale del aula. \\
\hline \multirow[t]{2}{*}{$\begin{array}{l}\text { P: Vamos muchacha. Habla alto para que } \\
\text { todo el mundo oiga. (Hablando con F8, } \\
\text { que está esperando para leer la actividad } \\
\text { realizada por el grupo 6.) }\end{array}$} & \\
\hline & $\begin{array}{l}\text { F8: Nuestro grupo piensa que todas las } \\
\text { personas deben ser tratadas de la misma } \\
\text { forma porque todas las personas son } \\
\text { iguales. }\end{array}$ \\
\hline $\begin{array}{l}\text { P: Principalmente esa parte ahí... todas } \\
\text { las personas son iguales... no se deben } \\
\text { hacer diferenciaciones entre las personas. } \\
\text { ¿Está bien? ¡Muy bien! Muchachos: } \\
\text { ¡Me gustó mucho! Algunas palabras clave } \\
\text { aparecieron... principalmente eso de } \\
\text { trato... prejuicio... que son palabras muy } \\
\text { importantes... fueron las dos principales } \\
\text { que oí. ¿Está bien? Y vamos a intentar } \\
\text { hacer eso más veces. ¿Está bien? ¡Muchas } \\
\text { gracias! (P mira para la filmadora y hace } \\
\text { un gesto de ok con el dedo, indicando la } \\
\text { finalización de la actividad.) }\end{array}$ & \\
\hline
\end{tabular}




\section{Hoja de registro de la actividad del Grupo 6:}

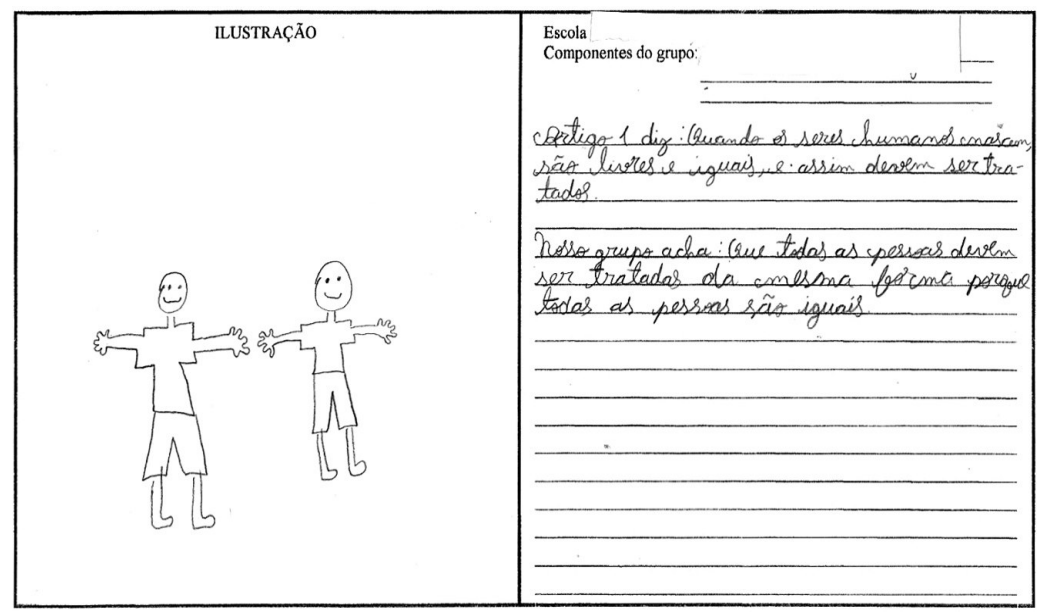

En este último techo del episodio, la presentación del grupo 6 es de la misma forma mecánica que las presentaciones anteriores. El profesor se contenta con la frase 'nuestro grupo piensa que todas las personas deben ser tratadas de la misma forma porque todas las personas son iguales', y encierra la discusión. Las reflexiones escritas y presentes en el dibujo de los niños no fueron aprovechadas por el profesor. No se discutieron aspectos relativos a los derechos humanos y moralidad. No fueron abordadas cuestiones como el concepto de igualdad, el significado de un trato igualitario en la vida cotidiana y su relación con la justicia.

La presentación del grupo 6 fue evaluada de manera simple (;Muy bien!), de la misma forma que la presentación de todos los grupos en la etapa final de la actividad estructurada: 'Muchachos: ¡Me gustó mucho! Algunas palabras clave aparecieron...'.

Las alumnas del grupo 6 se comprometieron con la actividad, pero sus reflexiones no surgieron de sus propias experiencias, pues se limitaron a copiar el artículo que leyeron. El dibujo podría ser más elaborado en función de la edad. Entre tanto, la forma de conducir la actividad indica que el dibujo y el texto escrito no tenían mucha importancia.

Fuente: Elaboración propia. Sesión de observación estructurada y filmada de la investigación.

De manera general, los resultados apuntaron la disponibilidad y motivación de los nińos para discutir y reflexionar sobre el tema propuesto, como ejemplifican los dos fragmentos presentados anteriormente. Específicamente, en el fragmento (5), presentación de la discusión y producción gráfica del grupo 4, los niños realizaron reflexiones amplias a partir de sus experiencias en relación con el artículo 11 de la DUDH, que no aborda directamente la posibilidad de acusaciones con base en prejuicios y venganza. De acuerdo con lo que fue colocado en el comentario analítico, esa reflexión no fue usada como punto de partida para discutir la cuestión con el grupo de manera más amplia; es 
decir, las concepciones y creencias de los nińos no fueron aprovechadas por el adulto, quien se limitó a felicitar a los dos grupos y a colocar sus propias creencias y valores en relación con los tópicos tratados. En ese momento, se dejaron de discutir temas importantes como el prejuicio, la igualdad y la justicia.

Los dos fragmentos seleccionados son representativos de la dinámica de la actividad estructurada planificada por el profesor. Esa actividad llegó al final sin alcanzar los objetivos pautados para ella; o sea, sin abrir un espacio de discusión y reflexión conjunta, por parte de los niños, sobre el tema de los DDHH. Los niños no tuvieron un espacio conjunto para discutir, reflexionar y expresarse de forma autónoma y creativa. La actividad pedagógica constituyó un espacio de reproducción de creencias y valores comunicados de manera asimétrica o unidireccional por el adulto, sin la posibilidad de protagonismo por parte de los alumnos.

El flujo de las interacciones indica que el adulto controló las acciones de los alumnos durante la actividad. De varias formas, el profesor enfatizó que los niños deberían realizar la actividad y participar según las reglas y planificación previamente establecidas por él. En relación con la importancia del protagonismo para la $\mathrm{EDH}$, sus directrices enfatizan la necesidad de pensar e implementar metodologías participativas que también permitan la construcción de las normas y reglas de organización de la dinámica escolar; en otras palabras, la participación activa de los alumnos no debe restringirse a la construcción del conocimiento. La participación efectiva de los niños en la organización social de su propio contexto educativo es un recurso fundamental para su proceso de socialización, para la construcción de valores sociales, morales y éticos que permitan la consolidación de una Cultura de Derechos Humanos (Ministério da Educação et al., 2013).

En este punto, es necesario destacar que el profesor organizó los grupos pequeños de trabajo considerando el sexo de los alumnos (tres grupos de niñas y tres grupos de niños). Esa división no se justifica ni por las características de la actividad realizada ni por la preferencia de los alumnos que no tuvieron la oportunidad de opinar sobre la organización de la dinámica. La interacción entre niños y niñas hubiera sido muy interesante en una discusión sobre los DDHH en función de las diferencias históricas de los derechos para hombres y mujeres. Por otro lado, la DUDH abarca artículos que establecen y resaltan la igualdad de derechos para todos.

Asimismo, es importante señalar que la actividad pedagógica siguió un patrón pregunta-respuesta-evaluación (P-R-E), es decir, el profesor preguntó para cada grupo sobre el artículo/contenido leído, los niños respondieron y sus respuestas fueron evaluadas con destaque para aquellas más importantes, de acuerdo con la visión del adulto. El patrón P-R-E es señalado como un 
patrón típico de interacciones asimétricas, en las cuales el profesor ejerce el papel social de control y de trasmisión del conocimiento. De acuerdo con Oliveira (1997), es un patrón característico de la interacción verbal de la educación tradicional, constituyéndose una estrategia discursiva que no abre espacio para la negociación y construcción conjunta de significados entre profesores y alumnos. El patrón P-R-E presupone que existe una respuesta correcta para la pregunta realizada por el profesor, y también presupone que profesores y alumnos tienen papeles específicos y restrictos en el contexto de las interacciones del proceso de enseñanza-aprendizaje. El profesor es aquel que sabe y el alumno necesita demostrar que sabe lo que el profesor espera de él. La tarea del profesor es evaluar y dar un retorno al alumno sobre su proceso de aprendizaje. Otra cuestión importante es que el patrón P-R-E no permite la alternancia entre los interlocutores de las actividades, siendo que esta última es una característica central del proceso de interacción verbal participativo (Oliveira, 1997). La falta de alternancia entre los interlocutores de la actividad estructurada fue evidente en todos los episodios delimitados y analizados, de la misma forma que aparece en los dos fragmentos presentados. Las interacciones, en el momento de la discusión grupal, se concentraron entre los alumnos portavoces de cada grupo pequeño y el profesor. Los otros alumnos participantes se limitaron a escuchar y esperar el momento de hablar.

Volviendo a las directrices de la EDH, y especialmente al Programa Mundial para la Educación en Derechos Humanos (Unesco, 2012), podemos decir que en la actividad pedagógica observada fue privilegiada la dimensión 'los derechos humanos en el contexto educativo', que destaca la importancia del aprendizaje de los DDHH a través de los planes de enseñanza y estudio, de los materiales didácticos y de los métodos pedagógicos y de capacitación. Entre tanto, ese aprendizaje de los DDHH no asumió un carácter histórico, ni tuvo lugar en un proceso participativo, de acuerdo con la resolución 1 de 30 de mayo de 2012 (Poder Executivo do Brasil, 2012). La complejidad de los procesos de construcción de valores sociales fue desconsiderada. Por otro lado, en el análisis de las interacciones que se establecieron entre los niños y el profesor (currículo oculto) es evidente que los contenidos relacionados a los DDHH fueron presentados a partir del modelo de transmisión unidireccional del conocimiento. En ningún momento, hubo una coconstrucción de nuevos elementos socioculturales por parte de los participantes, a partir de sus respectivos papeles sociales, de sus conocimientos y de su experiencia personal (Valsiner, 1989; 2007).

Aunque el aprendizaje de los DDHH sea una temática señalada como relevante por los educadores, todavía son cambios necesarios en el posicionamiento de los educadores delante de los procesos de enseñanza y aprendizaje. 
Como apuntamos en la introducción del presente trabajo, la EDH implica modificaciones que van más allá de los objetivos de la educación, abarcando las interacciones características del contexto escolar y el papel de la escuela en relación con la formación de creencias y valores éticos y morales (Tavares, 2007).

\section{Conclusiones}

De acuerdo con autores como Fernandes y Paludeto (2010), Klein y D’Água (2015) y Matos (2017), la educación que envuelve los DDHH todavía no forma parte de la práctica ni del currículo de la escuela brasileña, quedándose relegada a los textos legales. Aunque algunas escuelas realicen acciones para promover la EDH, esas acciones no hacen parte de una planificación intencional, sistematizada y consciente, plasmada en el Proyecto Político Pedagógico (PPP) de las instituciones. Por otro lado, no existe una reflexión sobre las prácticas pedagógicas y las interacciones/relaciones sociales del ambiente escolar, como escenario fundamental de la EDH. Ese panorama no es exclusivo de la escuela brasileña. En nivel internacional, algunos estudios, como el de Muńoz (2014) muestran las dificultades prácticas que surgen en la escuela a la hora de implementar proyectos de formación de valores para la convivencia.

Uno de los desafíos enfrentados por la escuela contemporánea está relacionado con el mantenimiento de un modelo tradicional y unidireccional de transmisión del conocimiento. Como se mencionó anteriormente, de acuerdo con dicho modelo, los significados compartidos socialmente deben ser transmitidos por personas con más experiencia, siendo papel del sujeto la asimilación pasiva de los contenidos trasmitidos con el intuito de adaptarse a la sociedad. El modelo enfatiza una relación asimétrica que no abre espacio para el posicionamiento activo del sujeto que 'recibe' la cultura. Siendo así, las prácticas pedagógicas pautadas con base en ese modelo limitan el protagonismo del alumno y, consecuentemente, la EDH y la educación para la ciudadanía, que deben tener como base la participación plena del individuo en todas las instancias y a partir de sus vivencias e historias sociales (Fernandes y Paludeto 2010). Tanto el Programa Mundial para la Educación en Derechos Humanos (Unesco, 2012) como el Plano Nacional de Educación en Derechos Humanos (Ministério da Educação e Secretaria Especial dos Direitos Humanos, 2006) señalan la importancia y necesidad de desarrollar una pedagogía participativa, promover la $\mathrm{EDH}$ como elemento relevante para los alumnos y fomentar discusiones sobre temas fundamentales para los DDHH.

Fernandes y Paludeto (2010) señalan que esas propuestas, leyes y acciones programáticas deben ser discutidas con los docentes, entendiendo que son ellos 
los actores de los cuales podemos obtener, o no, la adhesión necesaria para el desarrollo de un nuevo paradigma educativo. La formación de profesores para la EDH todavía es tímida, aunque los diversos sistemas de enseñanza y los centros de formación de profesores ya trabajen en esa perspectiva. La formación de profesores para la EDH, desafío importante del escenario educativo actual, debe contemplar tanto el conocimiento y análisis de sus propuestas como el análisis y cuestionamiento de las diferentes prácticas pedagógicas implementadas en la escuela. Asimismo, presupone un cambio en la visión del trabajo docente. Los profesores no deben ser vistos como 'técnicos' responsables por la enseñanza, sino como profesionales que pueden movilizar novedades en procesos personales y grupales de naturaleza cultural y social.

Sobre el análisis y cuestionamiento de las prácticas pedagógicas en la perspectiva de la $\mathrm{EDH}$, señalamos la necesidad de más investigaciones, específicamente en el contexto brasileño. De acuerdo con un estudio realizado por Bajerski (2015), la mayoría de las investigaciones realizadas en Brasil de 2011 a 2015 se centran en el tema de los DDHH de manera general. Existen pocos estudios sobre la $\mathrm{EDH}$ y las prácticas pedagógicas del contexto escolar, así como hay pocas investigaciones sobre la EDH y la formación de los educadores. Siendo así, también señalamos como especialmente importante la incrementación de estudios sobre la formación inicial de los profesores en la perspectiva de la EDH. Para Benevides, Amorim y Rego (2018), la EDH en la enseñanza superior es tratada de manera elemental y desarticulada en relación con los debates contemporáneos sobre los DDHH. Los diversos cursos de formación de docentes necesitan fortalecer sus prácticas educativas, incluyendo la EDH en el cotidiano de las instituciones de enseñanza superior.

\section{REFERENCIAS BIBLIOGRÁFICAS}

Bajerski, A. (2015). Análise bibliométrica da produção científica sobre educação e direitos humanos. Anais do XII Congresso Nacional de Educação (EDUCERE 2015), Curitiba, Brasil, 12. Recuperado de https://educere. bruc.com.br/arquivo/pdf2015/21218_10164.pdf

Barreto, A. M. R. F. (2004). Educação infantil: Crenças sobre as relaçôes entre práticas pedagógicas especificas e desenvolvimento da criança (Tese de Doutorado). Instituto de Psicologia da Universidade de Brasília, Brasília, Brasil.

Barrios, A. (2009). Desenvolvimento moral e práticas pedagógicas na educação infantil: Um estudo sociocultural construtivista (Dissertação de Mestrado). Programa de Pós-Graduação em Desenvolvimento Humano e Saúde, Universidade de Brasília, Brasília, Brasil. 
Barrios, A. (2013). Diálogo ou heteronomia no Ensino Fundamental? Desenvolvimento moral, cultura e práticas educativas (Tese de Doutorado). Programa de Pós-Graduação em Desenvolvimento Humano e Saúde, Universidade de Brasília, Brasília, Brasil.

Benevides, M., Amorim, R. y Rego, E. (2018). Educação em Direitos Humanos e Ensino Superior: Uma Análise do Currículo e da Formaçáo Docente nas Licenciaturas do Instituto Federal do Ceará. Contexto \& Educação, 33(104), 291-322. https://doi.org/10.21527/2179-1309.2018.104.291-322

Branco, A. (1989). Socialização na pré-escola: O papel da professora e da organizaçâo das atividades no desenvolvimento de interaçóes sociais entre crianças (Tese de Doutorado). Instituto de Psicologia da Universidade de São Paulo, SP, Brasil.

Branco, A. (2003). Social development in cultural context: Cooperative and competitive interaction patterns in peer relations. En J. Valsiner y K. J. Connolly (Orgs.), Handbook of Developmental Psychology (pp. 238-256). Londres: Sage. https://doi.org/10.4135/9781848608306.n11

Branco, A. y Mettel, T. (1995). O processo de canalização cultural das interações criança-criança na escola. Psicologia: teoria e pesquisa, 11(1), 13-22.

Branco, A., Barreto, A. R. y Barrios, A. (2018). Educational practices and young children's socio-moral development: a cultural psychological approach. En M. Fleer y B. V. Oers (Orgs.), International handbook of early childhood education (pp. 795-814). Dordrecht: Springer. https://doi. org/10.1007/978-94-024-0927-7_39

DeVries, R. y Zan, B. (1998). A ética na educação infantil: o ambiente sócio-moral na escola. Porto Alegre: Artes Médicas.

DeVries, R. y Zan, B. (2003). When children make rules. Educational Leadership: Building Classroom Relationship, 61(1), 64-67.

Fernandes, A. y Paludeto, M. (2010). Educação e Direitos Humanos: desafios para a escola contemporânea. Cadernos Cedes, 30(81), 233-249. https:// doi.org/10.1590/S0101-32622010000200008

Kelman, C. y Branco, A. (2004). Análise microgenética em pesquisa com alunos surdos. Revista Brasileira de Educação Especial, 10(1), 93-106.

Klein, A y D’Água, S. (2015). A educação em Direitos Humanos nos Projetos Políticos Pedagógicos das escolas de São Paulo. Educar em Revista, (55), 277-292. https://doi.org/10.1590/0104-4060.38443

Koga, V. y Rosso, A. (2016). Análise da produção científica acerca da educação moral na escola. Psicologia da Educação, 43(2), 47-57.

Lima, M. (2000). Silêncio elou participaçâa? A questão da disciplina na concepção e na prática de professoras da 1a. Série do Ensino Fundamental (Dissertação de Mestrado). Instituto de Psicologia da Universidade de Brasília, Brasília, Brasil. 
Lima, S. (2017). Indisciplina em sala de aula: concepçôes, interação social e ação docente - uma análise qualitativa. Atas do 6to. Congresso Ibero-americano de Investigação Qualitativa em Educação (CIAIQ 2017), Salamanca, España, 6. Recuperado de https://proceedings.ciaiq.org/index.php/ciaiq2017/ article/view/1404

Magendzo, A. (2006). Educación en derechos humanos: un desafio para los docentes de hoy. Santiago: LOM Ediciones.

Manzini, R. (2013). Bullying no contexto escolar: prevençấo da violência e promoção da cultura de paz na perspectiva de adultos e crianças (Tese de Doutorado). Programa de Pós-Graduação em Desenvolvimento Humano e Saúde, Universidade de Brasília, Brasília, Brasil.

Matos, F. (2017) Educação em Direitos Humanos: concep̧̧ôes e práticas educativas (Dissertação de Mestrado). Lisboa, Portugal: Instituto de Educação da Universidade Lusófona de Humanidades e Tecnologias.

Menin, M. (2002). Valores na escola. Educação e Pesquisa, 28(1), 91-100. https:// doi.org/10.1590/S1517-97022002000100006

Ministério da Educação e Secretaria Especial dos Direitos Humanos (2006). Programa Nacional de Educação em Direitos Humanos. Brasília: MEC.

Ministério da Educação; Secretaria de Educação Básica; Secretaria de Educação Continuada, Alfabetização, Diversidade e Inclusão; Secretaria de Educação Profissional e Tecnológica; Conselho Nacional de Educação e Câmara Nacional de Educação Básica (2013). Diretrizes curriculares nacionais para a Educação Básica. Brasília: MEC/SEF.

Muńoz, M. (2014). Diagnóstico e intervención en escuelas desde la política de convivencia escolar y enfoque de derechos humanos. Revista Electrónica "Actualidades Investigativas en Educación", 14 (3), 1-18. https://doi. org/10.15517/aie.v14i3.1609

Oliveira, M. (1997). Eventos interativos em sala de aula: estratégias discursivas do professor. Cadernos de Linguagem e Sociedade, 3(2), 84-99.

Palmieri, M. (2003). Cooperação, competição e individualismo: uma análise microgenética de contextos de desenvolvimento na pré-escola (Tese de Doutorado). Programa de Pós-Graduação em Desenvolvimento Humano e Saúde, Universidade de Brasília, Brasília, Brasil.

Poder Executivo do Brasil (2012) Resolução No. 1, de 30 de maio de 2012. Diário Oficial da República Federativa do Brasil, seção 1, p. 48. Brasília: Poder Executivo.

Ratner, C. (2002). Cultural Psychology: Theory and Method. Nueva York: Plenum. https://doi.org/10.1007/978-1-4615-0677-5 
Rogoff, B. (2005). A natureza cultural do desenvolvimento humano. Porto Alegre: Artmed.

Salomão, S. J. (2001). Motivação social: Comunicação e metacomunicação na coconstrução de crenças e valores no contexto de interaçóes professora-alunos (Dissertação de Mestrado). Instituto de Psicologia da Universidade de Brasília, Brasília, Brasil.

Silva, C. y Menin, M. (2015). Análise das relaçóes entre escola e comunidade em projetos de educação em valores. Anais da 41ts. Association for Moral Education Conference, Rio de Janeiro, Brasil, 41. Recuperado de http:// www.fecilcam.br/revista/index.php/anaisame/article/viewFile/1388/874

Tappan, M. B. (1992). Texts and contexts: Language, culture, and the development of moral functioning. En L. T. Winegar y J. Valsiner (Orgs.), Children's development within social context (pp. 93-117). Hillsdale, NJ: Lawrence Earlbaum Associates.

Tappan, M. B. (1997). Language, culture and moral development: A Vygotskian perspective. Developmental Review, 17(1), 78-100. https://doi. org/10.1006/drev.1996.0422

Tappan, M. B. (1998). Moral education in the zone of proximal development. Journal of Moral Education, 27(2), 141-160. https://doi.org/ $10.1080 / 0305724980270202$

Tavares, C. (2007). Educar em direitos humanos, o desafio da formaçáo dos educadores numa perspectiva interdisciplinar. En R. M. G. Silveira (Org.), Educação em Direitos Humanos: fundamentos teórico-metodológicos (pp. 487-503). João Pessoa: Editora Universitária.

Unesco (2012). Plano de Ação: Programa Mundial para Educação em Direitos Humanos (Primeira Fase). Brasília: Unesco.

Vallance, E. (1983). Hiding the hidden curriculum: An interpretation of the language of justification in Nineteenth Century Educational Reform. En H. Giroux y D. Purpel (Orgs.), The hidden curriculum and moral education: Deception or discovery? (pp. 9-27). Berkeley, CA: McCutchan Publishing Corporation.

Valsiner, J. (1989) Human development and culture: The social nature of personality and its study. Lexington, MA: Lexington.

Valsiner, J. (2007). Culture in minds and societies: Foundations of Cultural Psychology. Nueva Delhi: Sage.

Valsiner, J. (2012). Fundamentos da psicologia cultural: mundos da mente, mundos da vida. Porto Alegre: Artmed.

Vygotsky, L. S. (1984). A formação social da mente. Sáo Paulo: Martins Fontes.

Vygotsky, L. S. (2004). Psicologia pedagógica. São Paulo: Martins Fontes. 